\title{
Functionalization of Carbon-Hydrogen Bonds of Hydrocarbons and Ethers via Carbene Insertion
}

\author{
Ana Caballero, M. Mar Díaz-Requejo, Tomás R. Belderrain, M. Carmen Nicasio, \\ Swatioslaw Trofimenko, Pedro J. Pérez. \\ Departamento de Química y Ciencia de Materiales, Universidad de Huelva, Campus de \\ El Carmen, 21007-Huelva, Spain, and Department of Chemistry and Biochemistry, \\ University of Delaware, Newark, DE 19716
}

\section{Supporting Information}

Experimental and NMR data for compounds 4-18 
Table 1. Yields and regioselectivities for alkane activation products, 4-7.

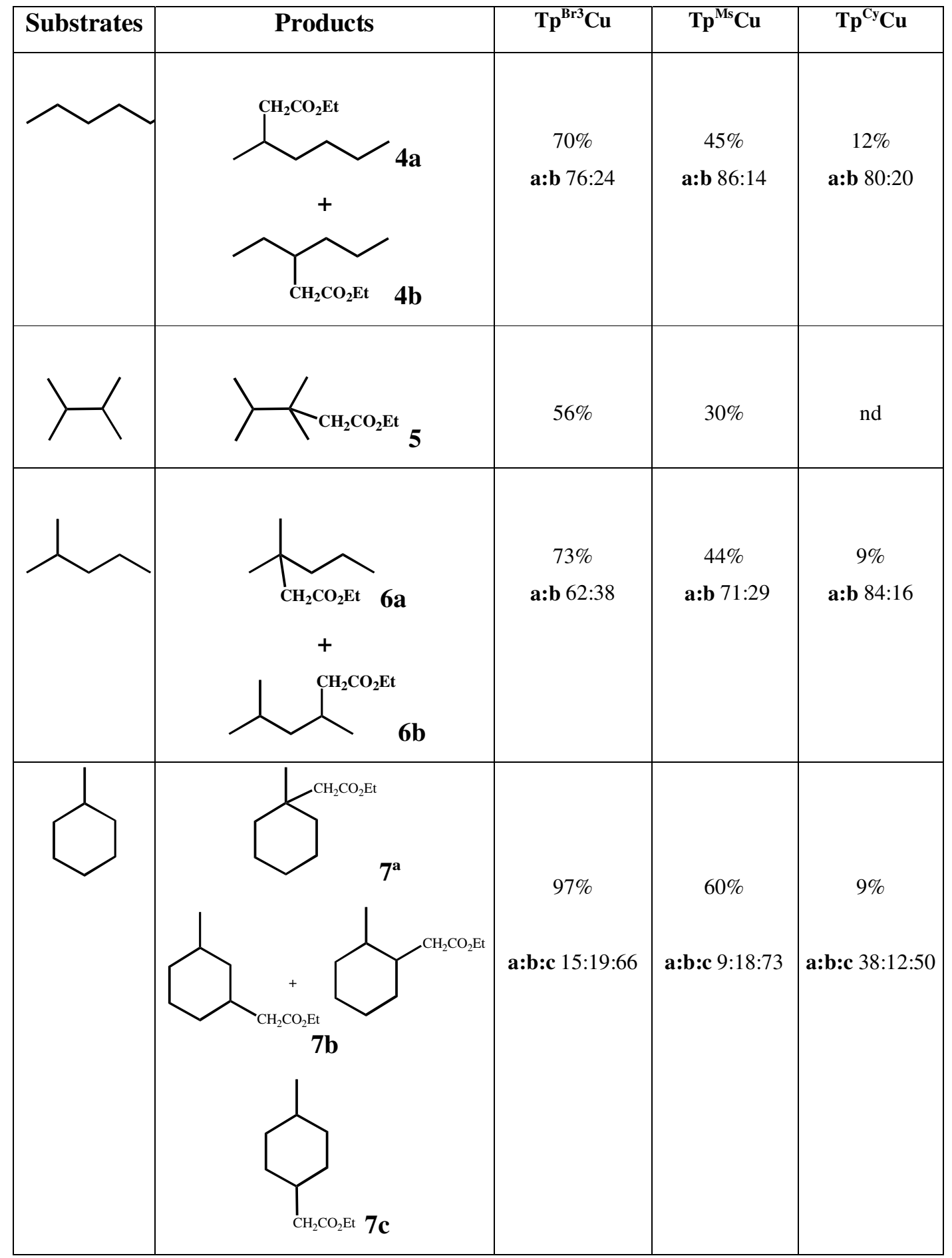

Yields and regioselectivities were calculated by GC analysis and/or by integration of the resonances corresponding to the olefin protons of fumarate and maleate, the $\mathrm{CH}_{2}$ of the ethoxy groups and the $\mathrm{RCH}_{2} \mathrm{CO}_{2} \mathrm{Et}$ of the products. 
Table 2. Yields and regioselectivities for cyclic ethers activation products, 8-12.

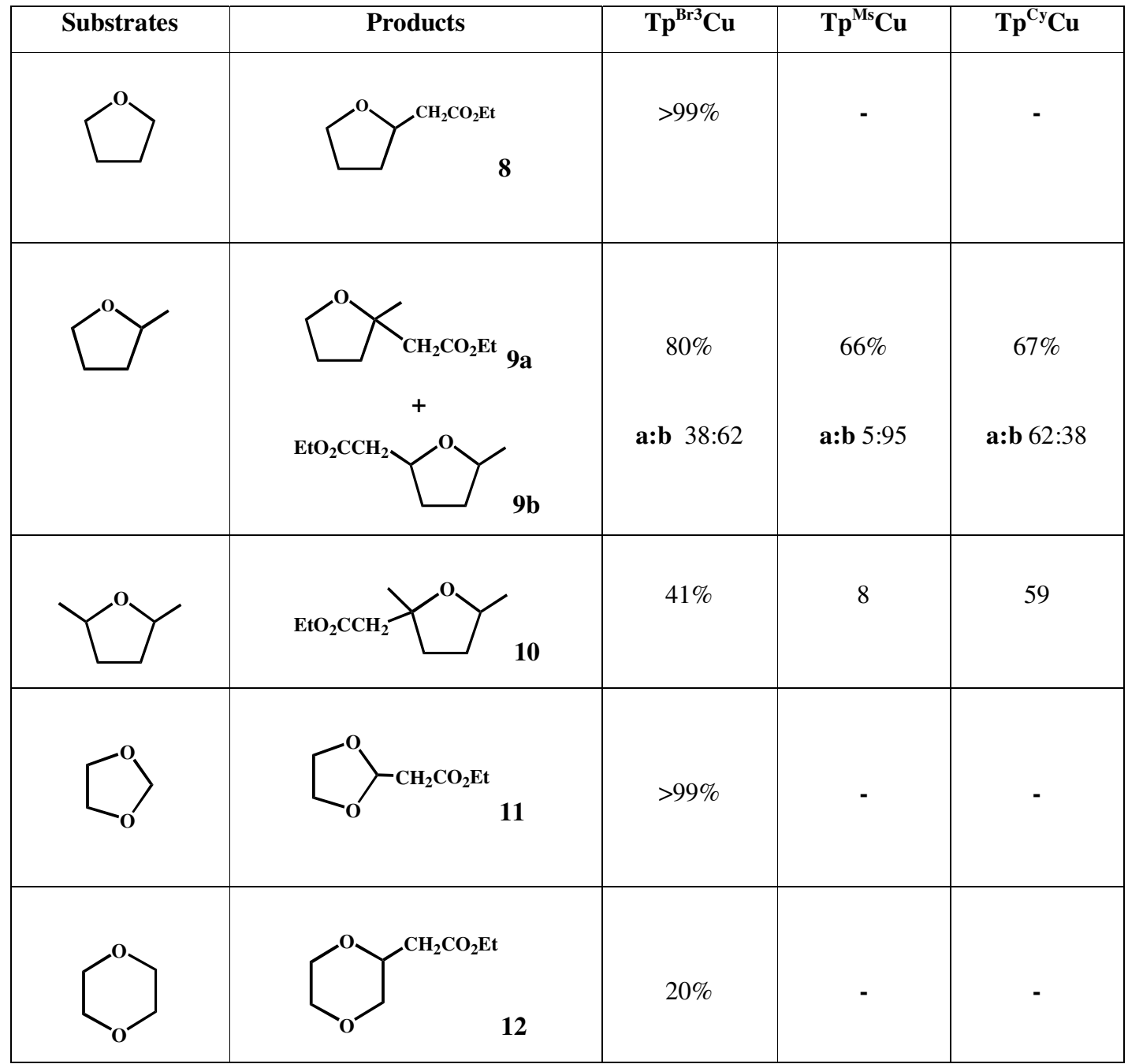

Yields and regioselectivities were calculated by GC analysis and/or by integration of the resonances corresponding to the olefin protons of fumarate and maleate, the $\mathrm{CH}_{2}$ of the ethoxy groups and the $\mathrm{RCH}_{2} \mathrm{CO}_{2} \mathrm{Et}$ of the products. 
Table 3. Yields and regioselectivities for ethers activation products, 12-18.

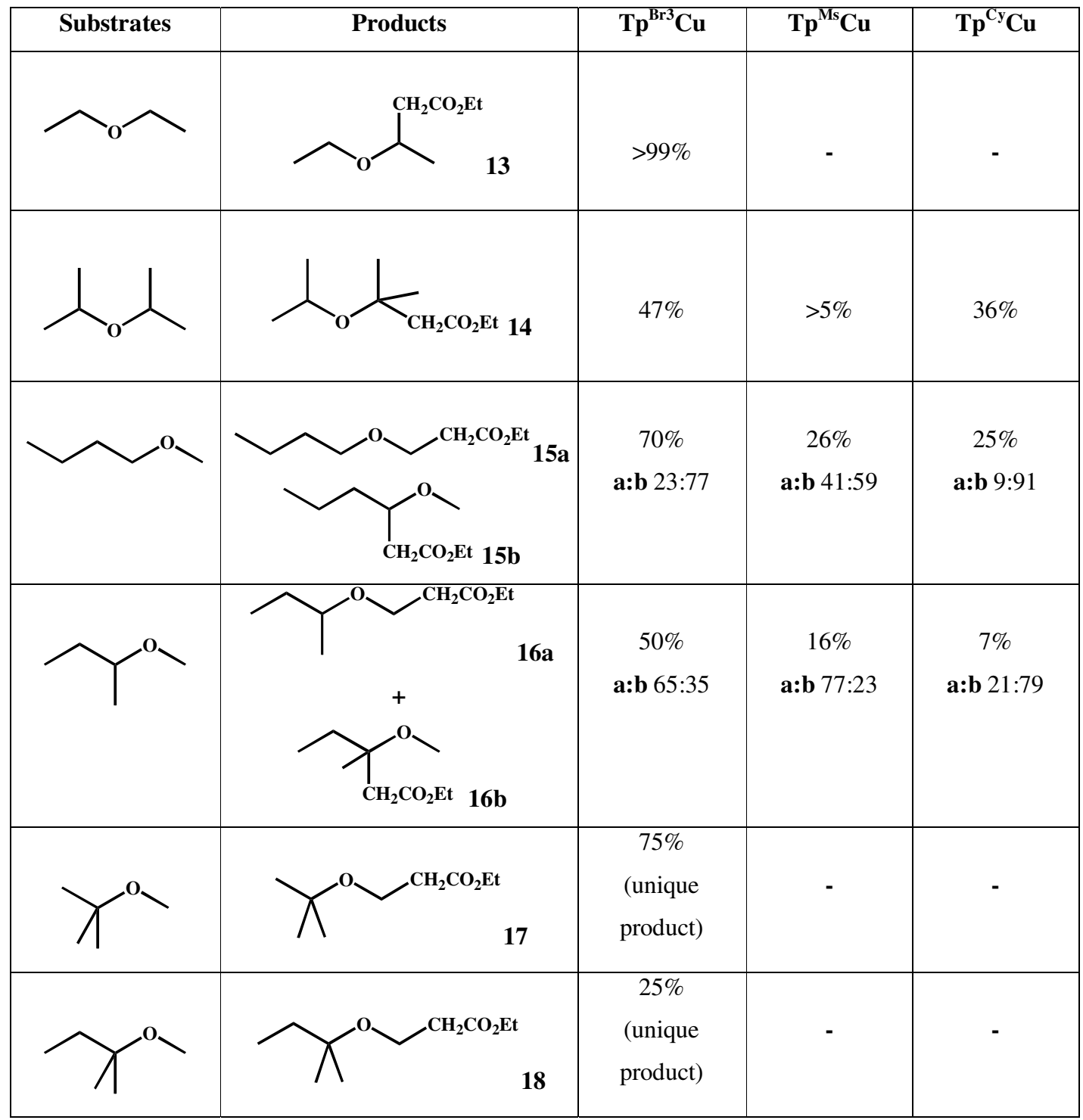

Yields and regioselectivities were calculated by GC analysis and/or by integration of the resonances corresponding to the olefin protons of fumarate and maleate, the $\mathrm{CH}_{2}$ of the ethoxy groups and the $\mathrm{RCH}_{2} \mathrm{CO}_{2} \mathrm{Et}$ of the products. 


\section{Reaction of EDA with alkanes}

$0.05 \mathrm{mmol}$ of $\mathrm{Tp} \mathrm{Cu}$ were disolved in a mixture of $\mathrm{CH}_{2} \mathrm{Cl}_{2}\left(\mathrm{~V}_{1}\right)$ and the alkane $\left(\mathrm{V}_{2}\right)$ (Table-S1). A solution of EDA $(1 \mathrm{mmol})$ in the alkane $(10 \mathrm{~mL})$ was slowly added for $\mathbf{t} \mathrm{h}$ with the aid of a syringe pump. No EDA was detected at the end of the reaction by GC.

Table S1. $\mathrm{V}_{1}, \mathrm{~V}_{2}$ and addition time, $t$, for each alkane.

\begin{tabular}{|c|c|c|c|}
\hline Alkane & $\mathbf{V}_{\mathbf{1}}(\mathbf{m l})$ & $\mathbf{V}_{\mathbf{2}}(\mathbf{m l})$ & $\mathbf{t} \mathbf{( h )}$ \\
\hline Hexane & 10 & 10 & 3 \\
\hline 2-methylbutane & 20 & 20 & 3 \\
\hline 2-methylpentane & 20 & 20 & 3 \\
\hline 2,3-dimethylbutane & 20 & 20 & 5 \\
\hline Methylcyclohexane & 5 & 15 & 3 \\
\hline
\end{tabular}

Ethyl 3-methylheptanoate (4a) and ethyl 3-ethylhexanoate (4b): Selected ${ }^{1} \mathrm{H}$ NMR (400 MHz, $\left.\mathrm{CDCl}_{3}\right)$ data for 4a: $\delta 2.08\left(\mathrm{dd}, 1 \mathrm{H},{ }^{2} J=14.6 \mathrm{~Hz},{ }^{3} J=8.0 \mathrm{~Hz}\right.$, diastereotopic $\left.\mathrm{RCH}_{2} \mathrm{CO}_{2} \mathrm{Et}\right)$ and $2.28\left(\mathrm{dd}, 1 \mathrm{H},{ }^{2} \mathrm{~J}=14.6 \mathrm{~Hz},{ }^{3} \mathrm{~J}=6.2 \mathrm{~Hz}\right.$, diastereotopic $\mathrm{RCH}_{2} \mathrm{CO}_{2} \mathrm{Et}$ ). Selected ${ }^{1} \mathrm{H}$ NMR $\left(400 \mathrm{MHz}, \mathrm{CDCl}_{3}\right.$ ) data for $\mathbf{4 b}: \delta 2.22\left(\mathrm{~d}, 2 \mathrm{H},{ }^{3} J=6.9\right.$ $\mathrm{Hz}, \mathrm{RCH}_{2} \mathrm{CO}_{2} \mathrm{Et}$ ). GC/MS for 4a: $\mathrm{m} / z$ (relative intensity) $173(\mathrm{M}+1), 157(\mathrm{M}-\mathrm{Me}), 115$ (M-Bu), $88\left(\mathrm{CH}_{2} \mathrm{CO}_{2} \mathrm{Et}+1\right)$. GC/MS for 4b: m/z (relative intensity) $173(\mathrm{M}+1), 143(\mathrm{M}-$ Et), 129 (M-Pr), $88\left(\mathrm{CH}_{2} \mathrm{CO}_{2} \mathrm{Et}+1\right)$.

Ethyl 3,3,4-trimethylpentanoate (5): Selected ${ }^{1} \mathrm{H}$ NMR (400 $\left.\mathrm{MHz}, \mathrm{CDCl}_{3}\right)$ data for 5.: $\delta 2.20\left(\mathrm{~s}, 2 \mathrm{H}, \mathrm{RCH}_{2} \mathrm{CO}_{2} \mathrm{Et}\right)$.

Ethyl 3,3-dimethylhexanoate (6a) and ethyl 3,5-dimethylhexanoate (6b): Selected ${ }^{1} \mathrm{H}$ NMR $\left(400 \mathrm{MHz}, \mathrm{CDCl}_{3}\right.$ ) data for $\mathbf{6 a}: \delta 2.17\left(\mathrm{~s}, 2 \mathrm{H}, \mathrm{RCH}_{2} \mathrm{CO}_{2} \mathrm{Et}\right.$ ). Selected ${ }^{1} \mathrm{H}$ NMR (400 MHz, $\mathrm{CDCl}_{3}$ ) data for $6 \mathbf{b}: \delta 2.08\left(\mathrm{dd}, 1 \mathrm{H},{ }^{2} J=14.3 \mathrm{~Hz},{ }^{3} J=8.1 \mathrm{~Hz}\right.$, diastereotopic $\left.\mathrm{RCH}_{2} \mathrm{CO}_{2} \mathrm{Et}\right)$ and $2.28\left(\mathrm{dd}, 1 \mathrm{H},{ }^{2} \mathrm{~J}=14.3 \mathrm{~Hz},{ }^{3} \mathrm{~J}=5.8 \mathrm{~Hz}\right.$, diastereotopic $\mathrm{RCH}_{2} \mathrm{CO}_{2} \mathrm{Et}$ ). GC/MS for 6a: $\mathrm{m} / z$ (relative intensity) $173(\mathrm{M}+1), 157(\mathrm{M}-\mathrm{Me}), 129(\mathrm{M}-$ Pr), $88\left(\mathrm{CH}_{2} \mathrm{CO}_{2} \mathrm{Et}+1\right)$. GC/MS for $\mathbf{6 b}: \mathrm{m} / z$ (relative intensity) $173(\mathrm{M}+1), 157(\mathrm{M}-\mathrm{Me})$, $115\left(\mathrm{M}-{ }^{\mathrm{i}} \mathrm{Bu}\right), 88\left(\mathrm{CH}_{2} \mathrm{CO}_{2} \mathrm{Et}+1\right)$. 
Ethyl ( $X$-methylcyclohexyl)acetate $(X=1,7 a ; X=2$ and $3,7 b ; X=4,7 c)$ : Selected ${ }^{1} \mathrm{H}$ NMR $\left(400 \mathrm{MHz}, \mathrm{CDCl}_{3}\right)$ data for $7 \mathbf{a}: \delta 1.02\left(\mathrm{~s}, 3 \mathrm{H}, \mathrm{CH}_{3}\right), 2.23(\mathrm{~s}, 2 \mathrm{H}$, $\left.\mathrm{RCH}_{2} \mathrm{CO}_{2} \mathrm{Et}\right)$. Selected ${ }^{1} \mathrm{H}$ NMR $\left(400 \mathrm{MHz}, \mathrm{CDCl}_{3}\right)$ data for $7 \mathrm{c}:: \delta: \delta 0.88\left(\mathrm{~d}, 3 \mathrm{H},{ }^{3} \mathrm{~J}=\right.$ $\left.6.5 \mathrm{~Hz}, \mathrm{CH}_{3}\right), 2.16\left(\mathrm{~d}, 2 \mathrm{H},{ }^{3} \mathrm{~J}=6.6 \mathrm{~Hz}, \mathrm{RCH}_{2} \mathrm{CO}_{2} \mathrm{Et}\right.$ ). Resonances of $\mathbf{7 b}$ isomers were small and overlapped by other peaks.

\section{Reaction of EDA with ethers}

$0.05 \mathrm{mmol}$ of $\mathrm{Tp}$ ' $\mathrm{Cu}$ were disolved in a mixture of $\mathrm{CH}_{2} \mathrm{Cl}_{2}\left(\mathrm{~V}_{1}\right)$ and the ether $\left(\mathrm{V}_{2}\right)$ (Table $\mathrm{S} 2$ ). A solution of EDA in $10 \mathrm{~mL}$ of $\mathrm{CH}_{2} \mathrm{Cl}_{2}$ (unless noted) was slowly added for $\mathbf{t} \mathrm{h}$ with the aid of a syringe pump. No EDA was detected at the end of the reaction by GC. After removal of volatiles, the crude product was investigated by ${ }^{1} \mathrm{H}$ NMR spectroscopy. Yields were calculated by integration of the resonances corresponding to the olefin protons of fumarate and maleate, the $\mathrm{CH}_{2}$ of the ethoxy groups and the $\mathrm{RCH}_{2} \mathrm{CO}_{2} \mathrm{Et}$ of the products.

Table S2. $\mathrm{V}_{1}, \mathrm{~V}_{2}$ and addition time, $t$, for each ether.

\begin{tabular}{|c|c|c|c|c|}
\hline Ether & EDA $(\mathbf{m m o l})$ & $\left.\mathbf{V}_{\mathbf{1}} \mathbf{( m l}\right)$ & $\left.\mathbf{V}_{\mathbf{2}} \mathbf{( m l}\right)$ & $\mathbf{t}(\mathbf{h})$ \\
\hline Tetrahydrofuran $^{\mathrm{a}}$ & 1 & - & 15 & 3 \\
\hline 2-methyltetrahydrofuran & 1 & 10 & 5 & 3 \\
\hline 2,5-dimethyltetrahydrofuran & 1 & 10 & 0.60 & 3 \\
\hline 2,2,5,5-tetramethyltetrahydrofuran & 1 & - & 10 & 3 \\
\hline Diethyl ether $^{\mathrm{a}}$ & 1 & - & 15 & 3 \\
\hline Isopropyl ether $^{\mathrm{b}}$ & 1 & 5 & 15 & 3 \\
\hline tert-butyl methyl ether $^{\mathrm{b}}$ & 5 & 10 & 0.12 & 3 \\
\hline tert-amyl methyl ether $^{\mathrm{b}}$ & 5 & 10 & 0.13 & 3 \\
\hline -butyl methyl ether $^{\text {sec-butyl methyl ether }}$ & 1 & 10 & 5 & 3 \\
\hline Dioxane $^{\mathrm{a}}$ & 1 & 10 & 5 & 3 \\
\hline Dioxolane $^{\mathrm{a}}$ & 1 & - & 15 & 2 \\
\hline
\end{tabular}

${ }^{\text {a }}$ Substrate used as solvent. ${ }^{\text {b }}$ A ratio substrate:EDA 1:5 is used.

Ethyl (tetrahydrofuran-2-yl)acetate (8): Selected ${ }^{1} \mathrm{H}$ NMR $\left(400 \mathrm{MHz}, \mathrm{CDCl}_{3}\right)$ data for 8: : $\delta 2.47\left(\mathrm{dd}, 1 \mathrm{H},{ }^{2} J=15.1 \mathrm{~Hz},{ }^{3} J=5.9 \mathrm{~Hz}\right.$, diastereotopic $\left.\mathrm{RCH}_{2} \mathrm{CO}_{2} \mathrm{Et}\right)$ and $2.59\left(\mathrm{dd}, 1 \mathrm{H},{ }^{2} \mathrm{~J}=15.1 \mathrm{~Hz},{ }^{3} \mathrm{~J}=7.2 \mathrm{~Hz}\right.$, diastereotopic $\left.\mathrm{RCH}_{2} \mathrm{CO}_{2} \mathrm{Et}\right)$. 
Ethyl (2-methyltetrahydrofuran-2-yl)acetate (9a) and Ethyl (5methyltetrahydrofuran-2-yl)acetate (9b): Selected ${ }^{1} \mathrm{H} \mathrm{NMR}\left(400 \mathrm{MHz}, \mathrm{CDCl}_{3}\right)$ data for 9a.: : $\delta 2.52\left(\mathrm{~d}, 1 \mathrm{H},{ }^{2} \mathrm{~J}=13.8 \mathrm{~Hz}\right.$, diastereotopic $\left.\mathrm{RCH}_{2} \mathrm{CO}_{2} \mathrm{Et}\right)$ and $2.54\left(\mathrm{~d}, 1 \mathrm{H},{ }^{2} \mathrm{~J}=\right.$ $13.8 \mathrm{~Hz}$, diastereotopic $\left.\mathrm{RCH}_{2} \mathrm{CO}_{2} \mathrm{Et}\right)$. . Selected ${ }^{1} \mathrm{H}$ NMR $\left(400 \mathrm{MHz}, \mathrm{CDCl}_{3}\right)$ data for 9b. (two stereoisomers): $\delta 2.42\left(\mathrm{dd}, 1 \mathrm{H},{ }^{2} J=15.1 \mathrm{~Hz},{ }^{3} J=6.4 \mathrm{~Hz}\right.$, diastereotopic $\mathrm{RCH}_{2} \mathrm{CO}_{2} \mathrm{Et}$ of major isomer), $2.48\left(\mathrm{~d}, 1 \mathrm{H},{ }^{2} \mathrm{~J}=15.2 \mathrm{~Hz},{ }^{3} \mathrm{~J}=6.5 \mathrm{~Hz}\right.$, diastereotopic $\mathrm{RCH}_{2} \mathrm{CO}_{2} \mathrm{Et}$ of minor isomer), $2.59\left(\mathrm{dd}, 1 \mathrm{H},{ }^{2} \mathrm{~J}=15.1 \mathrm{~Hz},{ }^{3} \mathrm{~J}=6.9 \mathrm{~Hz}\right.$, diastereotopic $\mathrm{RCH}_{2} \mathrm{CO}_{2} \mathrm{Et}$ of major isomer), $2.64\left(\mathrm{dd}, 1 \mathrm{H},{ }^{2} \mathrm{~J}=15.2 \mathrm{~Hz},{ }^{3} \mathrm{~J}=6.7 \mathrm{~Hz}\right.$, diastereotopic $\mathrm{RCH}_{2} \mathrm{CO}_{2} \mathrm{Et}$ of minor isomer). GC/MS for 9a: $\mathrm{m} / \mathrm{z}$ (relative intensity) $173(\mathrm{M}+1), 157$ (M-Me), $85\left(\mathrm{M}-\mathrm{CH}_{2} \mathrm{CO}_{2} \mathrm{Et}\right)$. GC/MS for 9b: $\mathrm{m} / z$ (relative intensity) $173(\mathrm{M}+1), 157$ $(\mathrm{M}-\mathrm{Me}), 130,85\left(\mathrm{M}-\mathrm{CH}_{2} \mathrm{CO}_{2} \mathrm{Et}\right)$.

Ethyl (2,5-dimethyltetrahydrofuran-2-yl)acetate (10): Selected ${ }^{1} \mathrm{H}$ NMR (400 $\mathrm{MHz}, \mathrm{CDCl}_{3}$ ) data for $\mathbf{1 0}$ (two stereoisomers): $\delta 2.53$ (s, $2 \mathrm{H}, \mathrm{RCH}_{2} \mathrm{CO}_{2}$ Et of major isomer), 2.62 (s, $2 \mathrm{H}, \mathrm{RCH}_{2} \mathrm{CO}_{2} \mathrm{Et}$ of minor isomer).

Ethyl 3-(dioxolan-2-yl)acetate (11): Selected ${ }^{1} \mathrm{H}$ NMR $\left(400 \mathrm{MHz}, \mathrm{CDCl}_{3}\right)$ data for 11: $\delta 2.65\left(\mathrm{~d}, 2 \mathrm{H},{ }^{3} J=5.2 \mathrm{~Hz}, \mathrm{RCH}_{2} \mathrm{CO}_{2} \mathrm{Et}\right), 3.9\left(\mathrm{t}, 1 \mathrm{H},,{ }^{3} J=5.2 \mathrm{~Hz}\right.$, $\left.\mathrm{CHCH}_{2} \mathrm{CO}_{2} \mathrm{Et}\right)$.

Ethyl 3-(dioxan-2-yl)acetate (12): Selected ${ }^{1} \mathrm{H}$ NMR (400 $\left.\mathrm{MHz}, \mathrm{CDCl}_{3}\right)$ data for 12: $\delta 2.28\left(\mathrm{dd}, 1 \mathrm{H},{ }^{2} J=15.5 \mathrm{~Hz},{ }^{3} J=5.8 \mathrm{~Hz}, \mathrm{RCH}_{2} \mathrm{CO}_{2} \mathrm{Et}\right.$ ), $2.43\left(\mathrm{dd}, 1 \mathrm{H},{ }^{2} J=15.5\right.$ $\left.\mathrm{Hz},{ }^{3} \mathrm{~J}=7.8 \mathrm{~Hz}, \mathrm{RCH}_{2} \mathrm{CO}_{2} \mathrm{Et}\right)$.

Ethyl 3-ethoxybutanoate (13).: Selected ${ }^{1} \mathrm{H}$ NMR $\left(400 \mathrm{MHz}, \mathrm{CDCl}_{3}\right)$ data for 13: $\delta 2.36\left(\mathrm{dd}, 1 \mathrm{H},{ }^{2} J=15.0 \mathrm{~Hz},{ }^{3} J=6.0 \mathrm{~Hz}\right.$, diastereotopic $\mathrm{RCH}_{2} \mathrm{CO}_{2} \mathrm{Et}$ ) and 2.57 (dd, $1 \mathrm{H},{ }^{2} \mathrm{~J}=15.0 \mathrm{~Hz},{ }^{3} \mathrm{~J}=7.0 \mathrm{~Hz}$, diastereotopic $\mathrm{RCH}_{2} \mathrm{CO}_{2} \mathrm{Et}$ ).

Ethyl 3-isopropoxy-3methylbutanoate (14): Selected ${ }^{1} \mathrm{H}$ NMR (400 MHz, $\mathrm{CDCl}_{3}$ ) data for 14: $\delta 2.48$ (s, $2 \mathrm{H}, \mathrm{RCH}_{2} \mathrm{CO}_{2} \mathrm{Et}$ ). GC/MS for 14: $\mathrm{m} / z$ (relative intensity) $189(\mathrm{M}+1), 173(\mathrm{M}-\mathrm{Me}), 101\left(\mathrm{M}-\mathrm{CH}_{2} \mathrm{CO}_{2} \mathrm{Et}\right) .145\left(\mathrm{M}-{ }^{\mathrm{i}} \mathrm{Pr}\right), 87\left(\mathrm{CH}_{2} \mathrm{CO}_{2} \mathrm{Et}\right), 59\left(\mathrm{O}^{\mathrm{i}} \mathrm{Pr}\right)$. 
Ethyl 3-butoxypropianoate (15a) and ethyl 3-metoxyhexanoate (15b): Selected ${ }^{1} \mathrm{H}$ NMR (400 MHz, $\mathrm{CDCl}_{3}$ ) data for 15a: $\delta 2.57\left(\mathrm{t}, 2 \mathrm{H},{ }^{3} J=6.2 \mathrm{~Hz}\right.$, $\mathrm{RCH}_{2} \mathrm{CO}_{2} \mathrm{Et}$ ), $3.58\left(\mathrm{t}, 2 \mathrm{H},{ }^{3} \mathrm{~J}=6.2 \mathrm{~Hz}, \mathrm{ROCH}_{2} \mathrm{CH}_{2} \mathrm{CO}_{2} \mathrm{Et}\right.$ ). Selected ${ }^{1} \mathrm{H}$ NMR (400 $\left.\mathrm{MHz}, \mathrm{CDCl}_{3}\right)$ data for 15b: $\delta 2.40\left(\mathrm{dd}, 1 \mathrm{H},{ }^{2} J=15.0 \mathrm{~Hz},{ }^{3} \mathrm{~J}=5.0 \mathrm{~Hz}\right.$, diastereotopic $\mathrm{RCH}_{2} \mathrm{CO}_{2} \mathrm{Et}$ ), $2.52\left(\mathrm{~d}, 1 \mathrm{H},{ }^{2} \mathrm{~J}=15.0 \mathrm{~Hz},{ }^{3} \mathrm{~J}=7.0 \mathrm{~Hz}\right.$, diastereotopic $\mathrm{RCH}_{2} \mathrm{CO}_{2} \mathrm{Et}$ ), 3.65 (m, $\left.1 \mathrm{H}, \mathrm{CHCH}_{2} \mathrm{CO}_{2} \mathrm{Et}\right)$.

Ethyl 3-sec-butoxypropionate (16a) and ethyl 3-methyl-3methoxypentanoate (16b): Selected ${ }^{1} \mathrm{H}$ NMR (400 $\left.\mathrm{MHz}, \mathrm{CDCl}_{3}\right)$ data for 16a: $\delta 2.55$ $\left(\mathrm{t}, 2 \mathrm{H},{ }^{3} \mathrm{~J}=6.6 \mathrm{~Hz}, \quad \mathrm{RCH}_{2} \mathrm{CO}_{2} \mathrm{Et}\right.$ ), 3.70 (two multiplets, $2 \mathrm{H}, A B$ system $\left.\mathrm{ROCH}_{2} \mathrm{CH}_{2} \mathrm{CO}_{2} \mathrm{Et}\right)$. Selected ${ }^{1} \mathrm{H}$ NMR $\left(400 \mathrm{MHz}, \mathrm{CDCl}_{3}\right)$ data for $\mathbf{1 6 b}: \delta 2.46(\mathrm{~d}, 1 \mathrm{H}$, ${ }^{2} J=13.0 \mathrm{~Hz}$, diastereotopic $\left.\mathrm{RCH}_{2} \mathrm{CO}_{2} \mathrm{Et}\right), 2.50\left(\mathrm{~d}, 1 \mathrm{H},{ }^{2} \mathrm{~J}=13.0 \mathrm{~Hz}\right.$, diastereotopic $\mathrm{RCH}_{2} \mathrm{CO}_{2} \mathrm{Et}$ ), 3.21 (s, $3 \mathrm{H}, \mathrm{CH}_{3} \mathrm{OR}$ ). GC/MS for 16a: $\mathrm{m} / \mathrm{z}$ (relative intensity) $175(\mathrm{M}+1)$, 159 (M-Me), 145 (M-Et), $117\left(\mathrm{M}-{ }^{\mathrm{s}} \mathrm{Bu}\right), 101\left(\mathrm{CH}_{2} \mathrm{CH}_{2} \mathrm{CO}_{2} \mathrm{Et}\right), 73\left(\mathrm{M}-\mathrm{CH}_{2} \mathrm{CH}_{2} \mathrm{CO}_{2} \mathrm{Et}\right)$, $57\left({ }^{\mathrm{s}} \mathrm{Bu}\right)$. ). GC/MS for 16b: $\mathrm{m} / \mathrm{z}$ (relative intensity) $175(\mathrm{M}+1), 159(\mathrm{M}-\mathrm{Me}), 145$ (M$\mathrm{Et}), 87\left(\mathrm{M}-\mathrm{CH}_{2} \mathrm{CO}_{2} \mathrm{Et}\right)$.

Ethyl 3-tert-butoxypropionate (17): Selected ${ }^{1} \mathrm{H} \mathrm{NMR}\left(400 \mathrm{MHz}, \mathrm{CDCl}_{3}\right)$ data for 17: $\delta 2.51\left(\mathrm{t}, 2 \mathrm{H},{ }^{3} J=6.6 \mathrm{~Hz}, \mathrm{RCH}_{2} \mathrm{CO}_{2} \mathrm{Et}\right), 3.62\left(\mathrm{t}, 2 \mathrm{H},,{ }^{3} J=6.6 \mathrm{~Hz}\right.$, ${ }^{\mathrm{t}} \mathrm{BuOCH} \mathrm{CH}_{2} \mathrm{CO}_{2} \mathrm{Et}$ ). GC/MS for 17: $\mathrm{m} / \mathrm{z}$ (relative intensity) $175(\mathrm{M}+1), 159(\mathrm{M}-\mathrm{Me})$, $117\left(\mathrm{M}-{ }^{\mathrm{t}} \mathrm{Bu}\right), 101\left(\mathrm{CH}_{2} \mathrm{CH}_{2} \mathrm{CO}_{2} \mathrm{Et}\right), 73\left(\mathrm{O}^{\mathrm{t}} \mathrm{Bu}\right), 57\left({ }^{\mathrm{t}} \mathrm{Bu}\right)$.

Ethyl 3-tert-pentoxypropionate (18): Selected ${ }^{1} \mathrm{H}$ NMR (400 $\left.\mathrm{MHz}, \mathrm{CDCl}_{3}\right)$ data for 18: $\delta 2.51\left(\mathrm{t}, 2 \mathrm{H},,{ }^{3} \mathrm{~J}=6.6 \mathrm{~Hz}, \mathrm{RCH}_{2} \mathrm{CO}_{2} \mathrm{Et}\right), 3.58\left(\mathrm{t}, 2 \mathrm{H},{ }^{3} \mathrm{~J}=6.6 \mathrm{~Hz}\right.$, $\mathrm{ROCH}_{2} \mathrm{CH}_{2} \mathrm{CO}_{2} \mathrm{Et}$ ). 